\title{
Infectious Lung Diseases and Endogenous Oxidative Stress
}

\author{
Kasturi Sarkar and Parames C. Sil
}

Abstract

Lower respiratory tract infections, according to the World Health Organization, account for nearly one third of all deaths from infectious diseases. They account for approximately 4 million deaths annually including children and adults and provide a greater disease burden than HIV and malaria. Among the common respiratory diseases, tuberculosis, influenza, and pneumonia are very common and can be life threatening if not treated properly. The causative agent of tuberculosis is the slow-growing bacilli Mycobacterium tuberculosis, while the causative agent of influenza is a segmented genome RNA virus. Pneumonia can be caused by a number of different microorganisms like bacteria, virus, and mycoplasma. In case of the entry of a pathogen in our body, the immune system gets activated, and the phagocytic cells try to eliminate it by generating reactive oxygen and nitrogen species (ROS and RNS) inside the phagosome. These reactive species or respiratory bursts are sufficient to eliminate most of the pathogens, except a few. M. tuberculosis is one such microorganism that has evolved mechanisms to escape this respiratory burst-mediated killing and thus survive and grow inside the macrophages. Infection with $M$. tuberculosis leads to the destruction of macrophages and release of cytokines, which lead to prolonged immune activation and oxidative stress. In some cases, the bacilli remain dormant inside macrophages for a long time. Flu viruses infect the epithelial cells present in respiratory tract, and the infection site is dependent on the hemagglutinin protein present on their capsid. Destruction of epithelial cells promotes secretion of mucus and activation of immune system leading to the oxidative damage. Community-acquired pneumonia is more serious and difficult to treat. In all

\author{
K. Sarkar \\ Department of Microbiology, St. Xavier's College, Kolkata, West Bengal, India \\ P. C. Sil $(\bowtie)$ \\ Division of Molecular Medicine, Bose Institute, Kolkata, West Bengal, India \\ e-mail: parames@jcbose.ac.in
}


these infections, ROS/RNS are developed as a defense mechanism against the pathogen. Persistence of the pathogen for a long time would lead to the uncontrolled production of ROS/RNS which will lead to oxidative stress and tissue damage to the host. Administration of antioxidants along with conventional treatments can be useful in the elimination of the reactive oxygen and nitrogen species.

\section{Keywords}

Tuberculosis · Influenza $\cdot$ Pneumonia $\cdot$ Macrophage $\cdot$ Neutrophil $\cdot$ Reactive oxygen species - Reactive nitrogen species · Respiratory burst - Oxidative stress · Antioxidants

\subsection{Introduction}

Respiratory infectious diseases are common problems among children and older people worldwide especially in developing countries. Nearly $20 \%$ of mortality in children under the age of 5 years is caused by these diseases. Most respiratory tract infections, especially the upper respiratory tract ones like rhinorrhea or pharyngitis, are mild and not incapacitating. However, lower respiratory tract infections like tuberculosis, flu, pneumonia, etc. can be more severe (Fitzpatrick et al. 2014). They are more likely to cause acute onset of fever, cough, dyspnea, or chest pain. Patients with respiratory infections should be diagnosed based on the disease features to expedite specific diagnosis and treatment.

This chapter is on three lower respiratory tract infections, viz., tuberculosis, influenza, and pneumonia, endogenous oxidative stress as a self-defense mechanism, and role of antioxidants on the infections.

Tuberculosis, an ancient infectious disease, can infect people of all age and almost every part of the human body. Tubercular lesions have been found on Neolithic skeletons (4500 BC) and on the bones of Egyptian mummies (1000 BC). The World Health Organization (WHO) declared that tuberculosis is among the top 10 causes of death worldwide, killing 1.6 million people in 2017 (Tuberculosis (TB) 2018). Among the worst affected countries are China, Southeast Asia, and the Indian subcontinent. The disease is most common in developing countries though the Centers for Disease Control and Prevention (CDC) reported over 9000 cases in the United States in 2016. Immunosuppression by stress or other illness, malnutrition, and poor hygienic condition activates the occurrence of tuberculosis (Sia et al. 2015).

Influenza, an acute respiratory infection, occurs in both pandemic and interpandemic forms. Over the past 100 years, global influenza pandemics have occurred approximately in every 10-30 years, whereas epidemic influenza occurs every winter in the temperate zones of both the hemispheres. Seasonal flu can be changed to acute respiratory illness with high mortality rate if converted to pandemic. The pathogenic H5N1 and H7N9 strains are currently in circulation and are a cause of great concern (Cline et al. 2017). Senior people, young children, and people with 
long-term lung diseases are more prone to serious complications from the flu. Flu can make asthma symptoms worse and can increase the risk of COPD flare-up.

Pneumonia can be caused by bacteria, viruses, and fungi or from inhalation of a chemical. Pneumonia can be life threatening and a leading cause of death and hospitalization in elder people and in people with other chronic diseases. People older than 65 or younger than 2 years of age or already having health problems are susceptible to pneumonia (Metersky et al. 2012).

\subsection{Cells in Alveoli}

There are three major types of cells present in the alveoli, viz., alveolar cells or pneumocytes and type I and II and alveolar macrophages (Jones, radiopaedia.org).

Type I Alveolar Cells or Pneumocytes are thin and flat and form the structure of alveoli. Type I cells take a major role in the gas exchange process between the alveoli and blood circulation. They are unable to replicate and sensitive to toxins. Type II alveolar cells are few in number $(<5 \%)$, and they are mostly found at the blood-air barrier and secrete surfactants. Type II cells play a major role to protect the lung in case of damage. They undergo cellular division, giving rise to more type I and II alveolar cells, without which the alveoli would collapse. Alveolar macrophages or the dust cells are very important in providing the lungs protection against microorganisms. They not only engulf the microorganisms, but they also scavenge any foreign molecules like dust, carbon particles, and molecules developed from blood cell injuries. There are three types of macrophages found in lungs. Alveolar macrophages or AMФs are found within the airways and guard the alveolar cells in bronchi, alveoli. The interstitial macrophages (IMФs) are found in lung interstitium, while the exudate-derived macrophages, EMФs, are recruited in response to an inflammation. These three populations are distinguishable from each other morphologically and functionally (https://epi.publichealth. nc.gov/cd/diseases/respiratory.html).

\subsection{Role of Macrophages and Neutrophils in Combating Infections}

The major phagocytic cells of our immune system are neutrophils, dendritic cells, and macrophages (Silva Manuel and Correia 2012) (Fig. 7.1). The phagocytic cells exhibit their microbicidal properties by generating huge amount of ROS and RNS within the phagosomes (Fang 2011). This metabolic process or respiratory burst is most prominent in neutrophils as compared to other phagocytic cells and causes oxidative stress. Respiratory burst initiates with the assembly of the components of an enzyme complex called NADPH oxidase (Groemping and Rittinger 2005) present on the plasma membrane and on the membranes of phagosomes in an inactive form. Upon phagocytosis of any molecule, assembly of the components leads to the formation of an active enzyme complex. This complex transfers one electron to $\mathrm{O}_{2}$ 


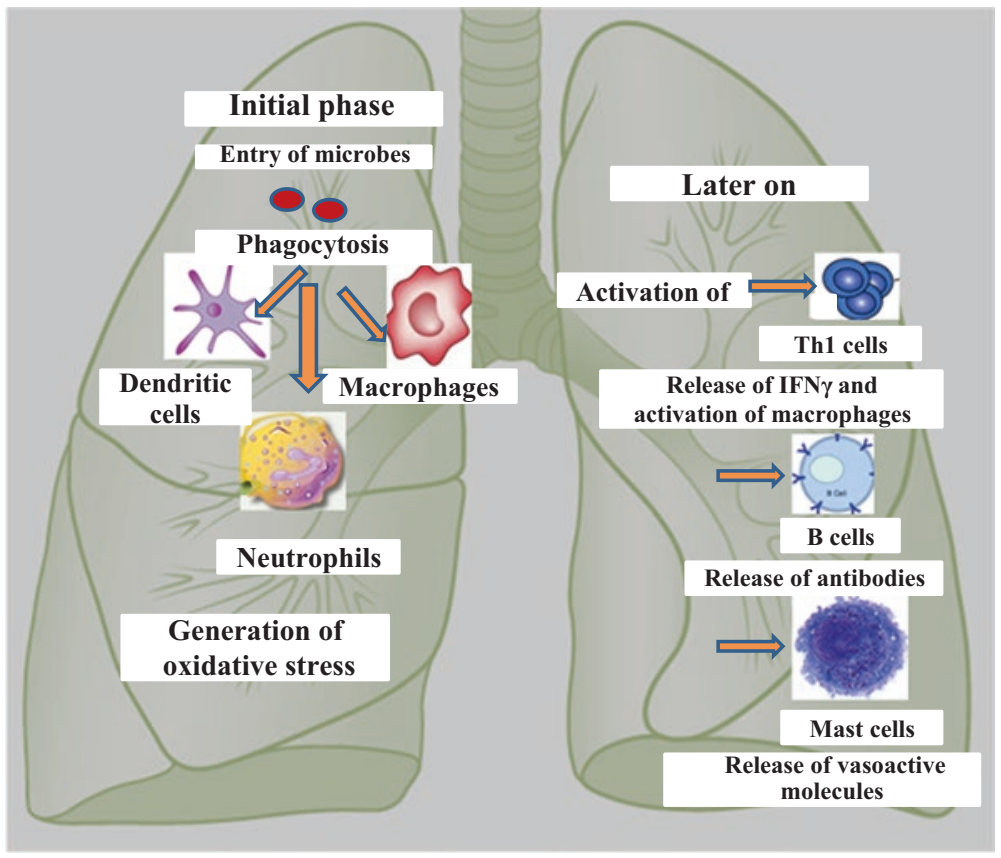

Fig. 7.1 Immune cells involved in fighting foreign invaders

from NADPH and forms superoxide radical $\left(\mathrm{O}_{2}{ }^{-}\right)$within the phagosomal lumen (Peterhans 1997). The superoxides can undergo spontaneous or enzyme-catalyzed dismutation to yield $\mathrm{H}_{2} \mathrm{O}_{2}$ and $\mathrm{O}_{2} \cdot \mathrm{H}_{2} \mathrm{O}_{2}$ combines with halides in presence of the enzyme myeloperoxidase and produces reactive hypohalous acid. These superoxide radicals may also interact with NO to produce peroxynitrite, ONOO-. These ROS/ RNS can damage a variety of biomolecules and kill the ingested microorganism (Halliwell and Aruoma 1991). They form adducts with unsaturated bonds and clusters having less electrons. Thus they can damage enzymes and unsaturated fatty acids or can oxidize $4 \mathrm{Fe}-4 \mathrm{~S}$ clusters present in enzymes or proteins resulting in metabolic defects. Damaged Fe-S clusters can release Fe, which will react with $\mathrm{H}_{2} \mathrm{O}_{2}$ (Fenton reaction) and yield $\mathrm{OH}$ leading to more damage. The cysteine residues present in proteins and enzymes can also interact with $\mathrm{H}_{2} \mathrm{O}_{2}$ directly. Superoxides do not damage membranes in bacteria, as they lack polyunsaturated fatty acids (Fig. 7.2).

Acidification of phagosomes is required for the optimal function of hydrolytic enzymes and thus for the degradation of the internalized pathogen. The acidification process is achieved by the recruitment of vacuolar proton-ATPase to the phagosomal membrane at the early phase of phagosome maturation. Recently it has been shown that TRPM2, the redox-sensitive transient receptor potential cation channel, expressed on the membrane of phagosome regulates the acidification and is essential for the efflux of $\mathrm{Ca}^{2+}$ during phagosome maturation (Di et al. 2017). 


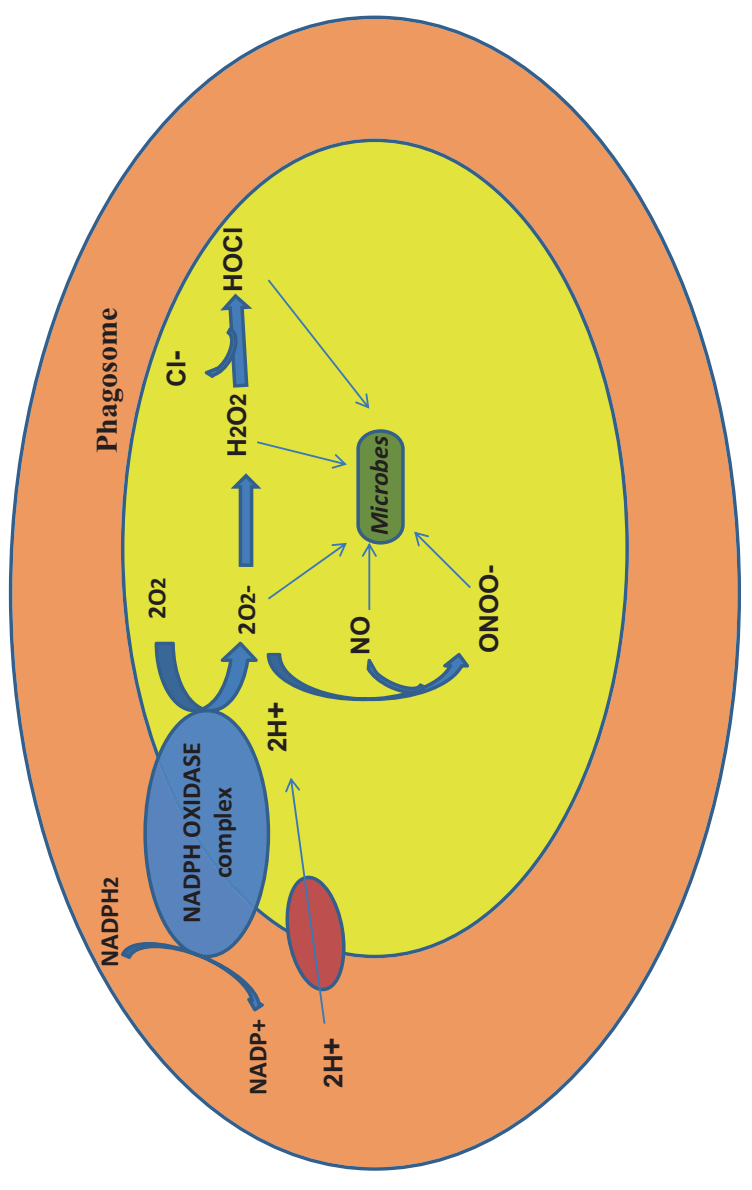

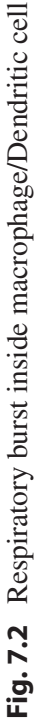


The antimicrobial capacity of neutrophils is higher than that of macrophages as their cytoplasm contains granules full of multiple antimicrobial molecules and peptides like defensins, cathelicidins, and proteins like lactoferrin and bactericidal/ permeability-increasing protein (Gudmundsson and Agerberth 1999).

\subsection{Effect of Excess ROS/RNS on Human Health}

The huge amounts of ROS generated as a defense mechanism against foreign molecules, if not removed adequately, will cause toxicity to all types of cells. It induces lipid peroxidation of host cell components, leading to the rise in intracellular calcium ions and DNA damage (Podinovskaia et al. 2013) (Fig. 7.3). Low levels of ROS influence the growth of some viruses like influenza, paramyxoviruses, HIV, etc. Chronic OS have been found to be associated with the development of cancer in case of some viral infections (Southwick 2007). Hence, antioxidants can play a major role in the treatment of viral diseases along by conventional drugs.

\subsection{Tuberculosis}

The highly aerobic and slow-growing microorganism Mycobacterium tuberculosis (Mtb) was discovered in 1882 by scientist Robert Koch. It can be identified with acid-fast stains or fluorescent stains under a microscope. The Mtb genome was sequenced in 1998 (Smith 2003) and has been found to have more than $99.9 \%$

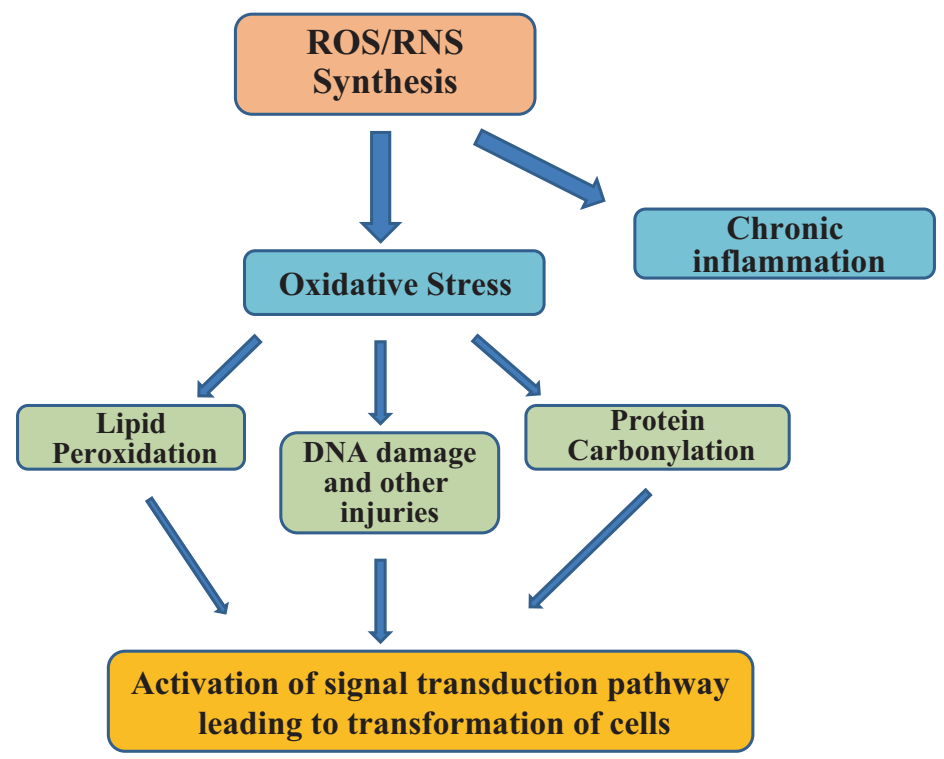

Fig. 7.3 Effect of oxidative stress on the host 
similarity with many other strains of Mycobacterium. The ability of Mtb to remain dormant in the host with the capacity to revive at a later time is because of its anaerobic persistency (Rook and Hernandez-Pando 1996).

\subsubsection{Symptoms of Tuberculosis}

Symptoms of tuberculosis are characterized by cough for 3 weeks or more, coughing up cloudy mucus, sometimes with blood, loss of weight, loss of appetite, weakness, fever, night sweats, sometimes shortness of breath, and chest pain (Tuberculosis (TB) 2018).

\subsubsection{Pathophysiology of Tuberculosis}

As discussed in the general section, macrophages kill Mtb by producing ROS/RNS in an oxygen-dependent manner. However, the bacterium has evolved mechanisms to survive inside the macrophages (Rook and Hernandez-Pando 1996). Depending on the nature and site of occurrence, TB is named differently.

Pulmonary Tuberculosis is characterized by the infection of the lung tissue by Mtb. The ROS/RNS produced by phagocytic cells restrict mycobacterial dissemination. A tuberculous lesion, called granuloma, is formed to confine the organism at the center where macrophages infected with Mtb are surrounded with noninfected phagocytes and T lymphocytes (Gengenbacher and Kaufmann 2012). Release of cytokines attracts more cells in the region, and sometimes macrophages fuse together to form multinucleated cells. In severe cases, the cellular granulomas are converted to necrotizing granulomas and lead to pulmonary lesions with extensive tissue damage. The core of the granuloma, called caseum, contains dead host cells and the bacilli (Gutsmann 2016).

Extrapulmonary Tuberculosis occurs because of the spreading of the infection outside the lungs to pleura, central nervous system, lymphatic system, genitourinary system, bone, joints, and other tissues. Mostly young people and people with weakened immune system are susceptible to this kind of spreading (Lee 2015).

Active Tuberculosis is characterized by TB symptoms and highly contagious. Hence, proper treatment should be followed by people suffering from active tuberculosis.

Latent Tuberculosis is characterized by the latency of Mtb inside the host. Most of the infected individuals with Mtb inside their macrophages don't develop the 
disease. Occurrence of the disease is dependent on two parameters, the virulence of the pathogen and the immune resistance of the host. The latent Mtb can become active at any time and needs proper treatment to cure the disease (Hauck et al. 2009).

\subsubsection{Entry of $M$. tuberculosis into Macrophages}

There are many molecules on the macrophage surface which act as receptors for Mtb like receptors for complement factors, carbohydrate moieties, Fc region of antibodies, etc. and intercellular adhesion molecules (Ernst 1998). The survival chance of Mtb depends on the receptor involved in phagocytosis. The entry of Mtb coated with specific antibodies and through receptors for antibody Fc regions would result in activation of respiratory burst in macrophages, whereas CR3 receptor-mediated entry doesn't lead to the activation of OS and thus increases the chance of survival of the bacterium (Caron and Hall 1998). Host plasma membrane cholesterol (Gatfield and Pieters 2000) has important roles to play in the entry of Mtb through phagocytosis and for the prevention of phagosome-lysosome fusion.

\subsubsection{Mechanisms by Which M. tuberculosis Escape the Macrophage-Mediated Killing}

- A macrophage membrane component, phosphatidylinositol 3-phosphate (PI3P), plays an important role in the phagosome maturation process. In Mtb-infected macrophages, Mtb glycolipids can block PI3P synthesis and thus interfere with phagosome-lysosome fusion and escape lysosome-mediated killing (Vergne et al. 2005).

- Another strategy is to prevent accumulation of PI3P on the membrane of phagosomes by degrading it by an acid phosphatase called SapM, a 28KDa protein, secreted by Mtb within the host cell cytosol (Saleh and Belisle 2000).

- Two protein phosphatases of Mtb, PtpA and B, interfere with host protein trafficking processes and increase the chance of survival for Mtb (Bach et al. 2008).

- PknG, another protein kinase of Mtb, prevents the degradation of Mtb in lysosomes (Scherr et al. 2007).

- The Mtb internalized phagosome is prevented from fusing with lysosomes by host protein coronin1/TACO and calcineurin and thereby helps in the survival of Mtb (Jayachandran et al. 2007).

- The proteasomes of Mtb neutralize the effect of NO and thus escape RNSmediated killing. In addition, the induction of the antimicrobial peptide cathelicidin (LL-37) is done through TLR signaling, and the process is vitamin D dependent (Fabri et al. 2011).

- Mtb produces several proteins/enzymes that are responsible for removal or detoxification of ROS and RNS. One such protein, KatG, a catalase peroxidase, destroys hydrogen peroxide. An extracellular Mn-Fe SOD (SodA) converts superoxide radical to $\mathrm{O}_{2}$ and $\mathrm{H}_{2} \mathrm{O}_{2}$. Another $\mathrm{Cu}-\mathrm{Zn}$ SOD (SodC) remains bound 
to the outer membrane of Mtb and possibly protects the surface against extracellular superoxides generated by host cells (Piddington et al. 2001).

- A genome-wide interaction study performed by Nambi et al. predicted the functional relationships among three proteins, the superoxide-detoxifying enzyme (SodA), an integral membrane protein (DoxX), and possibly a thiol oxidoreductase (SseA). This oxidoreductase complex helps Mtb to survive inside the host (Nambi et al. 2015).

\subsubsection{Oxygen-Independent Clearance of M. tuberculosis}

Upon entry into the system, Mtb are challenged to lung epithelial cells and macrophages (Rivas-Santiago et al. 2008). Infected bronchial epithelial cells produce defense molecules such as $\beta$-defensins, which directly kill the microbes by forming pores on their surface. In vitro infection of the lung epithelial cell line A549 (pneumocytes type II) with Mtb H37Rv induces the production of human beta defensin 2 (hBD-2) which is associated with mycobacterial lysis (Rivas-Santiago et al. 2005) Murine beta defensins 2 and 4 are expressed by the murine bronchial epithelium cells during the early phase of progressive pulmonary tuberculosis infection.

\subsubsection{Role of Neutrophils in Combating Mycobacterial Infections}

It has been found through studies that neutrophils are not very much engaged in combating Mtb but may play an important role by producing important cytokines and chemokines. Blomgran and Ernst showed that use of neutrophil-specific antibody (Ly6G-specific Ab 1A8) selectively depleted neutrophils and increased the frequency of dendritic cells (DC) in the lungs. They have concluded that neutrophils infected with Mtb secrete factors that attract DCs to facilitate interactions among these cells in the lungs (Blomgran and Ernst 2011).

\subsubsection{Role of Dendritic Cells Against M. tuberculosis}

There are controversies regarding the role of dendritic cells against Mtb. Some studies showed that dendritic cells, after phagocytosis of the pathogens, activate the $\mathrm{T}$ cells. Other reports showed that Mtb inhibits DC maturation and antigen presentation to T cells. Adane Mihret reported that infection of monocyte-derived dendritic cells (MDDCs) with Mtb leads to upregulation of major histocompatibility complex (MHC) proteins and other surface proteins like CD40, CD54, CD58, and CD80 which lead to effective presentation of the antigen to the T cells (Mihret 2012). 


\subsubsection{Tuberculosis and Oxidative Stress}

It is evident from numerous studies that changes in redox status of cells result in host cellular responses that will lead to proliferation or apoptosis or cell necrosis or mutation (Choi et al. 2009). This results in the damage of cell membrane resulting in fibrosis and dysfunction of lungs in pulmonary TB. Vijayamalini $\mathrm{M}$ et al. reported significant increase in lipid peroxidation marker, thiobarbituric acid reactive substance (TBARS), in pulmonary tuberculosis patients, both newly diagnosed and untreated, as compared to controls. The levels of antioxidants in plasma were found to decrease leading to the pathogenesis and severity of the disease (Vijayamalini and Manoharan 2004).

Generation of ROS increases in TB patients coinfected with HIV. Rajopadhye et al. reported decreased NO levels in serum of TB patients, while no change was observed in patients having both HIV and TB (Rajopadhye et al. 2017). There were increased levels of TBARS and CRP in both groups though increase in superoxide anion was found in HIV-TB group. Increased ROS production by the tat protein of HIV might activate NF-kB (Fiume et al. 2012) and lead to the increased rate of transcription in HIV. The activities of catalase and SOD were found to decrease in TB group. Increased SOD activity and total serum protein concentration were found in both groups compared to control. Power et al. reported in their study that antioxidant supplementation along with antituberculosis treatment (ATT) for 6 months significantly decreased the MDA level and increased the levels of vitamin $\mathrm{C}$ and $\mathrm{E}$ and total antioxidant status. Hence, their study concludes that supplementation of micronutrients or antioxidants promotes recovery of patients from tuberculosis by reducing OS (Pawar et al. 2011). Another work conducted by Janiszewska and group also reported that MDA concentration in the plasma of pulmonary tuberculosis patients was significantly higher before and after 1 and 2 months of antioxidant treatment with ATT compared to the control group which received only ATT (Janiszewska-Drobinska et al. 2001). Wagh et al. found in their study that the levels of prooxidants were significantly increased, whereas antioxidant markers were significantly decreased in the TB population compared to healthy controls (Wagh et al. 2016). In another experiment, the authors measured the OS markers and antioxidant capacity in granulomatous lesions in BCG-vaccinated and non-vaccinated guinea pigs infected with Mtb (Palanisamy et al. 2011). They observed decreased serum total antioxidant capacity and increased MDA concentration within lesions in nonvaccinated guinea pigs within 2 weeks of infection which were partially restored by the treatment of $\mathrm{N}$-acetyl cysteine. In addition, the antioxidant also decreased Mtb counts in the spleen and severity of lesion necrosis in the lung and spleen. Hence, proper antioxidants might be used as an adjunct therapy along with conventional ATT for better treatment and prevention of tuberculosis. 


\subsubsection{Diagnosis of TB}

Diagnosis of TB includes detection of presence of Mtb like Mantoux test (Nayak and Acharjya 2012), sputum smear test (Desikan 2013), and chest X-ray. New sensitive and rapid techniques include microscopic examination, microcolony detection, lipid profile analysis by HPLC, SDS-PAGE profiling to identify different strains of Mtb (Fatima 2009), amplification of the 16S-23S rDNA spacer region, etc.

\subsubsection{Antituberculosis Treatment (ATT)}

\subsubsection{Chemotherapy}

ATT includes first line of drugs like isoniazid, pyrazinamide, and ethambutol and drugs like fluoroquinolones, capreomycin, kanamycin, and amikacin. The secondline drugs include thioamides, cycloserine, and aminosalicylic acid. Other secondline drugs are clofazimine, amoxicillin with clavulanate, linezolid, carbapenems, etc. (Guidelines for treatment of tuberculosis 2010). Mismanagement of TB treatment can lead to resistance against multiple drugs called multidrug-resistant tuberculosis (MDR-TB) and extensively drug-resistant tuberculosis (XDR-TB).

To eliminate tuberculosis, WHO had introduced the Directly Observed Treatment Short (DOTS) course strategy which involved health workers to keep in touch with the TB patients for proper consumption of ATT drugs. In a report published in 1996, WHO claimed that DOTS was remarkably effective in some places, whereas it failed in sub-Saharan Africa (http://www.searo.who.int/tb/topics/what_dots/en/).

\subsubsection{Administration of Antioxidants Along with ATT}

It has been found by many researchers that the levels of antioxidants significantly lowered down in TB compared to control. The levels again rise during and after treatment of TB (Wiid et al. 2004). Reddy et al. supported the use of antioxidant supplementation for better improvement as an adjuvant therapy along with ATT (Reddy et al. 2004). Lamsal et al. observed similar results while treated TB patients with micronutrients along with ATT. Micronutrient treatment reduced the oxidant levels and increased antioxidant status (Lamsal et al. 2007). The supplementation also increased the production of IL-2 leading to the increased proliferation of T cells and decreased production of prostaglandin-2, T-cell suppressor. Kowalski et al. observed similar results in patients taking vitamins $\mathrm{C}$ and $\mathrm{E}$ (after 1-month ATT) (Kowalski et al. 2004). Studies had showed that there exists a potential supplemental role for micronutrient and other antioxidants in management of both drugsensitive and drug-resistant tuberculosis. The antioxidants may also have beneficial role in prevention and treatment of drug toxicity, particularly hepatotoxicity caused due to antitubercular drugs (Pawar et al. 2011; Verma et al. 2014). 


\subsubsection{Prevention}

A live vaccine is used for a long time which was derived from an attenuated strain of Mycobacterium bovis called the bacille Calmette-Guérin (BCG) vaccine (www. who.int/tb/challenges/hiv/07_tb_prevention_diagnosis_and_treatment_eng.pdf).

\subsection{Influenza}

There are four subtypes of influenza viruses, A, B, C, and D, which are very similar in overall structure. The common type, influenza A virus (IAV), infects aquatic birds, poultry animals, and humans. Influenza viruses have seven or eight pieces of -ve sense RNA as their genome which code for total 11 proteins present in the virus. HA, NA, and M2 are present on the envelope. There are about $18 \mathrm{HA}$ and 11 NA subtypes known, and all possible combinations are found in wild aquatic birds though HA 1, 2, and 3 and NA 1 and 2 are commonly found in humans.

\subsubsection{Symptoms}

Symptoms of flu include fever, cold sweats and shivers, headache, aching joints and limbs, fatigue, feeling of exhaustion, and gastrointestinal symptoms like nausea, vomiting, and diarrhea (http://www.who.int/news-room/fact-sheets/detail/ influenza-(seasonal)).

\subsubsection{Pathophysiology of Influenza}

Pathophysiology of influenza depends on the virulence of the flu virus and on host immune responses. Influenza virus can infect epithelial cells present both in upper and lower respiratory tract depending on the nature of the HA present on the capsid. In vivo studies showed that different IAV subtypes infect and replicate differently in the lung and thus result in different outcome (Taubenberger and Morens 2008). In most cases of mild and avirulent viruses, the HA is cleaved from the viral envelope by proteases in the upper respiratory tract, and these viruses mainly infect the nose, throat, and mouth. Hence, these strains are easily transmitted among people through sneezing and coughing. The HA of strains like $\mathrm{H} 5 \mathrm{~N} 1$ can be cleaved by a variety of proteases, allowing the virus to spread throughout the body. These strains cause severe flu but are not easily spread among people. The infected cells secrete huge amount of proinflammatory cytokines and chemokines that lead to inflammation. It is believed that the virus inhibits adrenocorticotropic hormone resulting in lowered cortisol levels (Taubenberger and Morens 2008). Coinfection of a susceptible cell by two IAVs at the same time can lead to antigenic shift where viral RNAs are mixed and a new shift virus strain can be generated. 


\subsubsection{Role of Macrophages in Removal of IAV}

IAV infects mainly the epithelial cells and alveolar macrophages (MФs) in the respiratory tract (Nicol and Dutia 2014). Tate et al. showed that viruses that readily infect $M \Phi s$ in vitro were less pathogenic in vivo than viruses with a limited ability to infect MФs (Tate Michelle et al. 2010). It has also been found that infection with IAV strains that replicate productively in MФs can induce many changes in M2 MФs, causing them to phenotypically resemble proinflammatory M1 MФs. Expression rate of the antibody Fc receptors (CD16 and CD32) present on MФs surface and responsible for opsonization has been found to decrease in IAV-infected cells (Cline et al. 2017).

Depletion of AMФs in mice and pigs using clodronate (released inside the MФs after phagocytosis and kills the cells)-loaded liposomes resulted in higher viral loads proving major role for MФs in controlling disease severity (Duan et al. 2017). Recent studies have shown that commensal microflora play an important role in the activation of pulmonary dendritic cells and induce specific immune responses against influenza virus (Samuelson et al. 2015).

\subsubsection{Role of Neutrophils on Influenza Virus}

Presence and increase in number of neutrophils in the airways is correlated with suppressed virus replication after flu infection (Camp and Jonsson 2017). Tate et al. established the critical role of neutrophils in the clearance of influenza virus by the use of specific monoclonal antibody (RB6-8C5) against them. Neutrophil depletion leads to increased number of virus particles and increased morbidity and mortality compared to control mice (Tate Michelle et al. 2008). Tumpey et al. established the role of macrophages and neutrophils in early-phase protection against an infection with a recombinant human influenza virus (H1N1) (Tumpey 2005). It can be inferred that neutrophils contributed to early protection against low inoculum of the virus but not against the high inoculums indicating a limitation of PMN function. Haruo Fujisawa showed that neutrophils provide protection against influenza viruses in tumor-bearing mice with neutrophilic leukocytosis (Fujisawa 2008). In vitro multiplication of influenza virus was also prevented by neutrophils from both normal and tumor-bearing mice. Addition of $N$-formylmethionyl-leucyl-phenylalanine (fMLP), a chemotactic factor and macrophage activator, to the culture, increased the inhibitory effect of neutrophils. Virus infectivity was found to decrease in the initial phase after infection in ICR and BALB/c mice though no such decrease was observed in tumor-bearing C57BU6 mice. Administration of fMLP inhibited the virus propagation in the lungs of normal and tumor-bearing mice having intranasal IAV infection. Hence, neutrophils play a significant role against IAV infection in vivo. 


\subsubsection{Influenza and Oxidative Stress}

High levels of proinflammatory cytokines, e.g., TNF- $\alpha$, IFN- $\beta$, CCL5, MIP-1 $\alpha$ (CCL3), MIP-1 $\beta$ (CCL4), MCP (CCL2), and IP-10 (CXCL10), have been found to be released by macrophages in humans infected with the highly pathogenic strain, H5N1 (Peschke et al. 1993). Infection with seasonal IAV also results in production of cytokines and chemokines from macrophages. The number of MФs increases in the lung in case of an IAV infection and returns to regular numbers following the resolution of the infection. Yu-Hsiang Lee et al. compared the immune responses induced by two strains of influenza virus, A/WSN/33 (H1N1) and A/Panama-like (H3N2) in C57BL/6 mice. In contrast to A/Panama-like (H3N2), WSN virus induced severe lung pathology accompanied by massive $\mathrm{Gr}-1^{+}$and $\mathrm{CD} 11 \mathrm{~b}^{+}$cell infiltration and high levels of CXCL6/GCP-2, CCL2/MCP-1, and TIMP-1 production. Both the Gr-1+ $1^{+}$and $\mathrm{CD} 11 \mathrm{~b}^{+}$cells produced ROS and RNS in lungs (Lee and Huang 2017). The level of xanthine oxidase, an enzyme synthesizing $\mathrm{O}^{2-}$, was found to increase in lung homogenates, and cells lavaged out of the lung showed a marked increase in $\mathrm{O}^{2-}$ production when stimulated with phorbol myristate acetate (PMA). In another model, Oda et al. showed that intravenously injected pyran copolymer-conjugated superoxide dismutase protected mice from the lethal effect of IAV (Oda et al. 1989). In addition to ROS, NO seems to play a role in the pathogenesis of IAV (Akaike et al. 2003).

Increased ROS production may contribute to an increase in the influenza virus titer (Belding et al. 1970). The reason lies on the effect of ROS on protease inhibitors present in the lung surfactant. The HA protein must be cleaved intracellularly into the dipeptides HA1 and HA2 for the propagation of the disease. Influenza virus strains that possess a HA that fits optimally with the intracellular protease is released into the extracellular space in the HA1/HA2 form, which is infectious. The HA that fits less optimally with the protease is released mostly in the noninfectious HA0 form (Kido et al. 1993). Cleavage of HA0 can still take place by the extracellular proteases present in pulmonary surfactant. As a protective mechanism, surface of alveoli carries antiproteases. These antiproteases can be inactivated by ROS. Hence, ROS generation can result into manyfold increase in infectious virus particles. A group of workers reported that intracellular ROS levels decrease in phagocytes after influenza infection. This decrease in ROS leads to increased susceptibility to secondary infections by bacteria like $S$. aureus (Sun et al. 2016a).

\subsubsection{Diagnosis}

Diagnosis of flu includes rapid influenza diagnostic test and rapid molecular assays (George 2012). 


\subsubsection{Treatment}

The two types of anti-influenza drugs used are inhibitors of neuraminidase (oseltamivir, zanamivir, laninamivir, and peramivir) and M2 protein (adamantane derivatives) (Boltz et al. 2010).

\subsubsection{Administration of Antioxidants Along with Conventional Treatment}

In a study $\mathrm{Xu}$ and Liu showed that treatment with curcumin after IAV infection downregulated productions of cytokines in a dose-dependent manner and inhibiting NF-кB signaling pathway (Xu and Liu 2017).

\subsubsection{Prevention}

The available trivalent vaccine protects against $\mathrm{H} 1 \mathrm{~N} 1$ and $\mathrm{H} 3 \mathrm{~N} 2$ and an influenza $\mathrm{B}$ virus. Another quadrivalent vaccine, available in market, provides protection against four flu viruses including an additional B virus.

\subsection{Pneumonia}

Bacterial Pneumonia is the most occurring pneumonia in all age groups. The common causative agent of community-acquired pneumonia (CAP) is the Gram+ve bacterium Streptococcus pneumoniae. Other Gram+ve organisms that cause pneumonia are Staphylococcus aureus and Bacillus anthracis. Among Gram-ve bacteria, Haemophilus influenzae, Klebsiella pneumoniae, Escherichia coli, Pseudomonas aeruginosa, Bordetella pertussis, and Moraxella catarrhalis are the frequent causative agents of pneumonia. Bacteria like Coxiella burnetii, Chlamydophila pneumoniae, Mycoplasma pneumoniae, and Legionella pneumophila also have been found to cause pneumonia. Yersinia pestis-caused pneumonia is usually called pneumonic plague. Nowadays drug-resistant Streptococcus pneumoniae (DRSP) and methicillin-resistant Staphylococcus aureus (MRSA) have become quite common (Nair and Niederman 2011).

Viral Pneumonia is caused by a number of viruses like rhinoviruses, coronaviruses, influenza viruses, respiratory syncytial viruses (RSVs), etc. Among them, RSVs are known to cause lung and airway infections in infants and young children more frequently. It is an enveloped virus with single-stranded RNA genome and belongs to the Pneumoviridae family of the Mononegavirales order (DawsonCaswell and Muncie Jr. 2011). 
Mycoplasma Pneumonia is the smallest and simplest self-limiting bacteria that cause mild upper respiratory tract infection to severe atypical pneumonia. M. pneumonia belongs to the class Mollicutes and family Mycoplasmataceae. They do not have cell wall and thus distinguished from bacteria. M. pneumoniae infections are transmitted via aerosols. Dorigo-Zetsma et al. genotyped and grouped clinical isolates of M. pneumoniae into eight subtypes based on P1 adhesin molecules (DorigoZetsma et al. 2000).

\subsubsection{Symptoms}

include cough sometimes with greenish or yellow mucus or bloody mucus, fever, shortness of breath, chest pain, headache, sweating, loss of appetite, low energy, and fatigue.

\subsubsection{Pathophysiology of Pneumonia}

Neutrophils remove the microorganisms and release cytokines that result in the activation of the immune system resulting in common symptoms like fever, chills, and fatigue. Sometimes elevated immune response leads to leakage in the blood capillaries in the lungs leading to plasma seepage into the alveoli resulting in a less functional area for gas exchange. Mucus plugs are released from the leaky capillaries into blood. WBCs accumulate in the lungs to clear the plugs, and eventually cell debris accumulate in the alveoli and make it solid, called consolidation, a feature of bacterial pneumonia. Unremoved bacteria can travel to the pleural cavity and blood stream from lungs and can result in sepsis and eventually septic shock, leading to damage in multiple organs (Eddy 2005).

\subsubsection{Macrophages Against Bacterial Pneumonia}

AMФs provide initial protection against bacterial pneumonia. However, when AMФs fail, neutrophils are predominantly recruited to control the infection (Dockrell et al. 2003). Aberdein et al. have shown that situations which lead to generation of less numbers of AMФs are major causes of development of pneumonia (Aberdein et al. 2013). S. pneumoniae is not killed by ROS as the bacterium has developed several mechanisms to escape OS-mediated killing. They are eliminated by NO and peroxynitrite, which are produced by macrophages in response to pneumococcal cell wall and the toxin pneumolysin (MacMicking et al. 1997). 


\subsubsection{Neutrophils Against Bacterial Pneumonia}

It is known for a long time that lower respiratory tract bacterial infection causes appearance of morphologically immature neutrophils in the circulation resulting increased synthesis and release of neutrophils from the marrow. Experiments have proved that selective depletion of the neutrophils results in profound defects in the clearance of bacteria like $S$. pneumoniae, K. pneumoniae, and L. pneumophila from the lungs. Neutrophils secrete serine proteases like cathepsin G and elastase which mediate the effective killing of ingested S. pneumoniae (Marriott et al. 2008). Investigations have also shown that efficient neutrophil accumulation is important to induce a successful adaptive immune response in the host.

\subsubsection{Macrophages Against RSV}

The role of alveolar macrophages was studied by Philippa and coworkers in acute respiratory RSV infection by depleting macrophages by the intranasal administration of clodronate liposomes in an established mouse model (Pribul Philippa et al. 2008). The decreased concentrations of local inflammatory cytokines and chemokines, less number of macrophages and natural killer cells, and enhanced viral load in the lung proved that macrophages play a central role in the early responses to viral infection. However, macrophages have been found to have little effect on the adaptive response as there was no change in the number of $\mathrm{T}$ cells.

\subsubsection{Role of Neutrophils in RSV Infection}

Neutrophils play a major role in RSV infection and lead to a strong systemic immune response. They have been found to produce the enzyme elastase and express activation markers like CD11b, CD18, and CD54 on their surface in response to RSV. The amount of neutrophil response is dependent on the clinical severity and viral load. The presence of viral genome and mRNA inside neutrophils suggests phagocytosis or replication of virions within neutrophils (Stokes et al. 2013).

\subsubsection{Immune Cells Against Mycoplasma}

Neutrophils isolated from the airway infected with mycoplasma have been found to contain high amount of histamine compared to naive neutrophils. Mycoplasma has been found to directly stimulate the expression of mRNA encoding histidine decarboxylase, an enzyme required in histamine synthesis in vitro. Treatment with antihistamines in in vivo showed decreased severity of pneumonia and tracheobronchitis in infected mice (Xu et al. 2006). Mast cells also lead to allergic inflammation by the release of histamine in mycoplasma infection. A toxin called 
community-acquired respiratory distress syndrome toxin (CARDS) produced by $M$. pneumonia promotes the generation of functional $\mathrm{IgE}$ in mice. Thus $M$. pneumoniaeinduced infections are strongly associated with asthma and its exacerbations (Medina et al. 2017).

\subsubsection{Community-Acquired Pneumonia (CAP) and Oxidative Stress}

Oxidative stress plays a crucial role in the development and progression of community-acquired pneumonia, the most common infectious illness. It was revealed that five times higher $\mathrm{H}_{2} \mathrm{O}_{2}$ is released in exhaled air of CAP patients than control and the amount decreases with treatment. The authors suggested that the sources of $\mathrm{H}_{2} \mathrm{O}_{2}$ were activated leucocytes, monocytes, and macrophages, and development of OS leads to the activation of neutrophils and other effector cells with generation of excess active oxygen forms in the lungs of CAP patients (Majewska et al. 2004). These ROS migrate through the alveolar-capillary membrane in the process of gas exchange and are able to induce the OS development in the erythrocytes (Ugurlu 2016). Trefler in their work showed increased levels of TBARS in CAP caused by bacteria compared to control. However, they observed lower TBARS levels and increased glutathione redox system in viral CAP caused by H1N1 compared to normal (Trefler et al. 2014). In another study, Vilen et al. showed that there was significant increase in advanced oxidation protein products (AOPP) and MDA in blood plasma of CAP patients compared to control group though there was no change in the concentration of reactive protein carbonyl derivatives (Molotov-Luchanskiy et al. 2015). Muravlyova showed that parameters like erythrocyte aggregation, total oxidant status, and OS index increased in IIP patients than controls, whereas some parameters like erythrocyte deformability, PV, and total antioxidant status remain unaltered (Muravlyova et al. 2016). It has been found that postinfluenza Staphylococcus aureus pneumonia leads to extensive lung inflammation even after antibiotic treatment. The chronic granulomatous diseases in humans are linked to X chromosome-linked Nox2 expression (Sun et al. 2016b). Yuanyuan Chen et al. demonstrated increase in OS and cytokines like TNF- $\alpha$ and IL-6 in the lung and peripheral blood with increase in the severity of CAP. Vitamin C supplementation inhibited ROS, DNA damage, TNF- $\alpha$, and IL-6 in LPSstimulated macrophages. It also inhibited autophagy in MH-S cells exposed to LPS and $\mathrm{H}_{2} \mathrm{O}_{2}$ (Chen et al. 2014). Rodrigo et al. observed lower FRAP and the GSH/ GSSG ratio and increased lipid peroxidation in both plasma and erythrocytes in CAP than control values. Thus the antioxidant status alterations are correlated with clinical severity (Castillo et al. 2013). Many studies have showed that administration of antioxidants such as Vitamins $\mathrm{E}$ and $\mathrm{C}$ have positive effects on oxidative biomarkers in in vivo and in vitro models of CAP (Siempos et al. 2008). There are controversies regarding the use of vitamins in CAP though (Merchant et al. 2004). 


\subsubsection{Diagnosis}

Diagnosis of pneumonia is based on physical examinations, chest radiography, CT scan, and laboratory tests. Laboratory tests include leukocyte count, sputum Gram stain, blood cultures, pulse oximetry, and urine antigens. Recent studies open up the possibility of using OS indicators as diagnostic tools for CAP severity as well as for the estimation of effectiveness of CAP treatment (Pavlyshyn et al. 2014).

\subsubsection{Treatment}

Treatment of pneumonia includes antibiotics to treat bacterial pneumonia and with fever and/or pain relievers (Donovan 2018).

\subsubsection{Prevention}

Prevention of pneumonia is provided by pneumococcal conjugate vaccines against Streptococcus pneumoniae which are effective in children. Development of new and improved vaccines and awareness among people would lead to further improvement in strategies for the prevention of CAP.

\subsection{Conclusion}

The common lower respiratory tract infectious diseases like tuberculosis, influenza, and pneumonia can be life threatening if not treated properly. The host mechanism to fight with the microorganisms is mainly by generating ROS and RNS by the immune cells. Chronic infections and persistence of microorganisms inside the host lead to uncontrolled activation of immune system and generation of reactive species, which eventually cause enormous damage to the host system. Several studies have showed that the deleterious effects of the oxidative stress can be fought with the administration of antioxidants along with regular treatment regimen.

\section{References}

Aberdein JD, Cole J, Bewley MA, Marriott HM, Dockrell DH (2013) Alveolar macrophages in pulmonary host defence - the unrecognized role of apoptosis as a mechanism of intracellular bacterial killing. Clin Exp Immunol 174(2):193-202

Akaike T, Okamoto S, Sawa T, Yoshitake J, Tamura F, Ichimori K, Miyazaki K, Sasamoto K, Maeda H (2003) 8-Nitroguanosine formation in viral pneumonia and its implication for pathogenesis. PNAS 100(2):685-690

Bach H, Papavinasasundaram GK, Wong D, Hmama Z, Av-Gay Y (2008) Mycobacterium tuberculosis virulence is mediated by PtpA dephosphorylation of human vacuolar protein sorting 33B. Cell Host Microbe 3:316-322 
Belding ME, Klebanoff SJ, Ray CG (1970) Peroxidase-mediated virucidal systems. Science 167(3915):195-196

Blomgran R, Ernst JD (2011) Lung neutrophils facilitate activation of naïve antigen specific CD4+ T cells during Mycobacterium tuberculosis infection. J Immunol 186(12):7110-7119

Boltz DA, Aldridge JR Jr, Webster RG, Govorkova EA (2010) Drugs in development for influenza. Drugs 70(11):1349-1362

Camp JV, Jonsson CB (2017) A role for neutrophils in viral respiratory disease. Front Immunol 8:550. https://doi.org/10.3389/fimmu.2017.00550. eCollection 2017

Caron E, Hall A (1998) Identification of two distinct mechanisms of phagocytosis controlled by different Rho GTPases. Science 282:1717-1721

Castillo RL, Carrasco RA, Álvarez PI, Ruiz M, Luchsinger V, Zunino E, Martínez MA, Avendaño LF (2013) Relationship between severity of adult community-acquired pneumonia and impairment of the antioxidant defense system. Biol Res 46(2):207-213

Chen Y, Luo G, Yuan J, Yuanyuan W, Xiaoqiong Y, Xiaoyun W, Guoping L, Zhiguang L, Nanshan Z (2014) Vitamin C mitigates oxidative stress and tumor necrosis factor-alpha in severe community-acquired pneumonia and LPS-induced macrophages. Mediat Inflamm. https://doi. org/10.1155/2014/426740. Article ID 426740, 11 page

Choi K, Kim J, Kim GW, Choi C (2009) Oxidative stress-induced necrotic cell death via mitochondria-dependent burst of reactive oxygen species. Curr Neurovasc Res 6(4):213-222

Cline TD, Beck D, Bianchini E (2017) Influenza virus replication in macrophages: balancing protection and pathogenesis. J Gen Virol 98(10):2401-2412

Dawson-Caswell M, Muncie HL Jr (2011) Respiratory syncytial virus infection in children. Am Fam Physician 83(2):141-146

Desikan P (2013) Sputum smear microscopy in tuberculosis: is it still relevant? Ind J Med Res 137(3):442-444

Di A, Kiya T, Gong H, Gao X, Malik AB (2017) Role of the phagosomal redox-sensitive TRP channel TRPM2 in regulating bactericidal activity of macrophages. J Cell Sci 130(4):735-744

Dockrell DH, Marriott HM, Prince LR et al (2003) Alveolar macrophage apoptosis contributes to pneumococcal clearance in a resolving model of pulmonary infection. J Immunol $171: 5380-5388$

Donovan FM (2018) Chief Editor: Thomas E Herchline. Community-acquired pneumonia empiric therapy. Drugs \& diseases infectious diseases. Updated: Jul 25

Dorigo-Zetsma JW, Dankert J, Zaat SA (2000) Genotyping of Mycoplasma pneumoniae clinical isolates reveals eight P1 subtypes within two genomic groups. J Clin Microbiol 38:965-970

Duan M, Hibbs ML, Chen W (2017) The contributions of lung macrophage and monocyte heterogeneity to influenza pathogenesis. Immunol Cell Biol 95(3):225

Eddy O (2005) Community-acquired pneumonia: from common pathogens to emerging resistance. Emerg Med Pract 7(12):1-22

Ernst J (1998) Macrophage receptors for mycobacterium tuberculosis. Infect Immun 66(4):1277-1281

Fabri M et al (2011) Vitamin D is required for IFN- $\gamma$-mediated antimicrobial activity of human macrophages. Sci Transl Med 3(104):104ra102

Fang FC (2011) Antimicrobial actions of reactive oxygen species. MBio 2(5):e00141-e00111. https://doi.org/10.1128/mBio.00141-11

Fatima N (2009) Newer diagnostic techniques for tuberculosis. Respir Med CME 2(4):151-154

Fitzpatrick ME, Sethi S, Daley C, Ray P, Beck JB, Gingo MR (2014) Infections in "Noninfectious" lung diseases. Ann Am Thorac Soc 11(4):S221-S226

Fiume G, Vecchio E, De Laurentiis A, Trimboli F, Palmieri C, Pisano A et al (2012) Human immu-

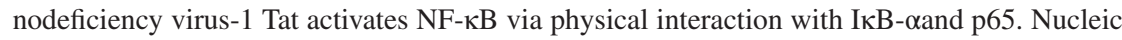
Acids Res 40(8):3548-3562

Fujisawa H (2008) Neutrophils play an essential role in cooperation with antibody in both protection against and recovery from pulmonary infection with the influenza virus in mice. J Virol 82(6):2772-2783 
Gatfield J, Pieters J (2000) Essential role for cholesterol in entry of mycobacteria into macrophages. Science 288(5471):1647-1650

Gengenbacher M, Kaufmann SHE (2012) Mycobacterium tuberculosis: success through dormancy. FEMS Microbiol Rev 36(3):514-532

George KS (2012) Diagnosis of influenza virus. Methods Mol Biol 865:53-69. https://doi. org/10.1007/978-1-61779-621-0_4

Groemping Y, Rittinger K (2005) Activation and assembly of the NADPH oxidase: a structural perspective. Biochem J 386(Pt 3):401-416

Gudmundsson GH, Agerberth B (1999) Neutrophil antibacterial peptides, multifunctional effector molecules in the mammalian immune system. J Immunol Methods 232(1-2):45-54

Guidelines for treatment of tuberculosis Fourth edition. Authors: World Health Organization. Publication date: 2010, ISBN: 9789241547833 WHO reference number: WHO/HTM/ $\mathrm{TB} / 2009.420$

Gutsmann T (2016) Interaction between antimicrobial peptides and mycobacteria. Biochim Biophys Acta Biomembr 1858(5):1034-1043

Halliwell B, Aruoma OI (1991) DNA damage by oxygen-derived species its mechanism and measurement in mammalian systems. FEBS Lett 281(1-2):9-19

Hauck FR, Neese BH, Panchal AS (2009) Identification and management of latent tuberculosis infection. Am Fam Physician 79(10):879-886

http://www.searo.who.int/tb/topics/what_dots/en/

http://www.who.int/news-room/fact-sheets/detail/influenza-(seasonal)

https://epi.publichealth.nc.gov/cd/diseases/respiratory.html

Janiszewska-Drobinska B, Kowalski J, Blaszczyk J, Kedziora J, Kaczmarek P, Cie wierz J (2001) Estimation of plasma malonyl dialdehyde concentration in patients with pulmonary tuberculosis. Pol Merkur Lekarski 11(64):310-313

Jayachandran R, Sundaramurthy V, Combaluzier B, Mueller P, Korf H, Huygen K, Miyazaki T, Albrecht I, Massner J, Pieters J (2007) Survival of mycobacteria in macrophages is mediated by Coronin 1-dependent Activation of Calcineurin. Cell 130(1):37-50

Jones J. Bronchopulmonary segmental anatomyl radiology reference article|Radiopaedia.org. radiopaedia.org

Kido H, Sakai K, Kishino Y, Tashiro M (1993) Pulmonary surfactant is a potential endogenous inhibitor of proteolytic activation of Sendai virus and influenza A virus. FEBS Lett 322(2): $115-119$

Kowalski J, Janiszewska-Drobinska B, Pawlicki L, Ceglinski T, Irzmanski R (2004) Plasma antioxidative activity in patients with pulmonary tuberculosis. Pol Merkur Lekarski 16(92):119-122

Lamsal M, Gautam N, Bhatta N, Toora BD, Bhattacharya SK, Baral N (2007) Evaluation of lipid peroxidation product, nitrite and antioxidant levels in newly diagnosed and two months followup patients with pulmonary tuberculosis. Southeast Asian J Trop Med Public Health 38(4):695-703

Lee JY (2015) Diagnosis and treatment of Extrapulmonary tuberculosis. Tuberc Respir Dis (Seoul) 78(2):47-55

Lee Y-H, Huang J-H (2017) Mucosa-associated lymphoid tissue lymphoma translocation protein 1 positively modulates matrix Metalloproteinase-9 production in alveolar macrophages upon toll-like receptor 7 signaling and influenza virus infection. Front Immunol 8:1177

MacMicking J, Xie QW, Nathan C (1997) Nitric oxide and macrophage function. Annu Rev Immunol 15:323-350

Majewska E, Kasielski M, Luczynski R et al (2004) Elevated exhalation of hydrogen peroxide and thiobarbituric acid reactive substances in patients with community acquired pneumonia. Respir Med 98:669-676. https://doi.org/10.1016/j.rmed.2003.08.015

Marriott HM, Jackson LE, Wilkinson TS, Simpson AJ, Mitchell TJ, Buttle DJ, Cross SS, Ince PG, Hellewell PG, Whyte MK, Dockrell DH (2008) Reactive oxygen species regulate neutrophil recruitment and survival in pneumococcal pneumonia. Am J Respir Crit Care Med 177(8):887-895 
Medina JL, Brooks EG, Chaparro A, Dube PH (2017) Mycoplasma pneumoniae CARDS toxin elicits a functional IgE response in Balb/c mice. PLoS One. https://doi.org/10.1371/journal. pone. 0172447

Merchant AT, Curhan G, Bendich A, Singh VN, Willett WC, Fawzi WW (2004) Vitamin intake is not associated with community-acquired pneumonia in U.S. men. J Nutr 134(2):439-444

Metersky ML, Masterton RG, Lode H, File TD Jr, Babinchak T (2012) Epidemiology, microbiology, and treatment considerations for bacterial pneumonia complicating influenza. Int J Infect Dis 16:e321-e331

Mihret A (2012) The role of dendritic cells in Mycobacterium tuberculosis infection. Virulence 3(7):654-659

Molotov-Luchanskiy V, Muravyova L, Bakirova R, Klyuyev D, Demidchik L, Kolesnikova Y (2015) Biomarkers for oxidative stress in patients with community-acquired pneumonia. Eur Respir J 46(59)

Muravlyova L, Molotov-Luchankiy V, Bakirova R, Klyuyev D, Demidchik L, Lee V (2016) Characteristic of the oxidative stress in blood of patients in dependence of community-acquired pneumonia severity. Open Access Maced J Med Sci 4(1):122-127. https://doi.org/10.3889/ oamjms. 2016.040

Nair GB, Niederman MS (2011) Community-acquired pneumonia: an unfinished battle. Med Clin North Am 95(6):1143-1161

Nambi S, Long JE, Mishra BB, Baker R, Murphy KC, Olive AJ, Nguyen HP, Shaffer SA, Sassetti CM (2015) The oxidative stress network of Mycobacterium tuberculosis reveals coordination between radical detoxification systems. Cell Host Microbe 17(6):829-837

Nayak S, Acharjya B (2012) Mantoux test and its interpretation. Indian Dermatol Online J 3(1):2-6

Nicol MQ, Dutia BM (2014) The role of macrophages in influenza A virus infection. Futur Virol 9(9):847-862

Oda T, Akaike T, Hamamoto T, Suzuki F, Hirano T, Maeda H (1989) Oxygen radicals in influenza-induced pathogenesis and treatment with pyran polymer-conjugated SOD. Science 244(4):974-976

Palanisamy GS, Kirk NM, Ackart DF, Shanley CA, Orme IM (2011) Evidence for oxidative stress and defective antioxidant response in Guinea pigs with tuberculosis. PLoS One 6(10):e26254

Pavlyshyn H, Sarapuk I, Slyva V, Haliyash N (2014) PO-1029 diagnostic value of apoptotic activity and oxidative stress in community-acquired pneumonia in infants. Arch Dis Child 99(Suppl 2):A587-A587

Pawar BD, Suryakar AN, Khandelwal AS (2011) Effect of micronutrients supplementation on oxidative stress and antioxidant status in pulmonary tuberculosis. Biomed Res 22(4):455-459

Peschke T, Bender A, Nain M, Gemsa D (1993) Role of macrophage cytokines in influenza A virus infections. Immunobiology 189(3-4):340-355

Peterhans E (1997) Oxidants and antioxidants in viral diseases: disease mechanisms and metabolic regulation. J Nutr 127(5):962S-965S. https://doi.org/10.1093/jn/127.5.962S

Piddington DL, Fang FC, Laessig T, Cooper AM, Orme IM, Buchmeier NA (2001) Cu, Zn superoxide dismutase of Mycobacterium tuberculosis contributes to survival in activated macrophages that are generating an oxidative burst. Infect Immun 69(8):4980-4987

Podinovskaia M, Lee W, Caldwell S, Russell DG (2013) Infection of macrophages with Mycobacterium tuberculosis induces global modifications to phagosomal function. Cell Microbiol 15(6):843-859

Pribul Philippa K, James H, Belinda W, Hongwei W, Tregoning John S, Jurgen S, Openshaw Peter JM (2008) Alveolar macrophages are a major determinant of early responses to viral lung infection but do not influence subsequent disease development. J Virol 82(9):4441-4448

Rajopadhye SH, Mukherjee SR, Chowdhary AS, Dandekar SP (2017) Oxidative stress markers in tuberculosis and HIV/TB co-infection. J Clin Diagn Res 11(8):BC24-BC28

Reddy YN, Murthy SV, Krishna DR, Prabhakar MC (2004) Role of free radicals and antioxidants in tuberculosis patients. Indian J Tuberc 51:213-218 
Rivas-Santiago B, Schwander SK, Sarabia C (2005) Human \{beta\}-defensin 2 is expressed and associated with Mycobacterium tuberculosis during infection of human alveolar epithelial cells. Infect Immun 73:4505-4511

Rivas-Santiago B, Contreras JCL, Sada E, Hernández-Pando R (2008) The potential role of lung epithelial cells and $\beta$-defensins in experimental latent tuberculosis. Scand J Immunol 67(5):448-452

Rook GAW, Hernandez-Pando R (1996) The pathogenesis of tuberculosis. Annu Rev Microbiol 50:259-284

Saleh MT, Belisle JT (2000) Secretion of an acid phosphatase (SapM) by Mycobacterium tuberculosis that is similar to eukaryotic acid phosphatases. J Bacteriol 182(23):6850-6853

Samuelson DR, Welsh DA, Shellito JE (2015) Regulation of lung immunity and host defense by the intestinal microbiota. Front Microbiol 6:1085

Scherr N, Honnappa S, Kunz G, Mueller P, Jayachandran R, Winkler F, Pieters J, Steinmetz MO (2007) Structural basis for the specific inhibition of protein kinase G, a virulence factor of Mycobacterium tuberculosis. Proc Natl Acad Sci U S A 104(29):12151-12156

Sia JK, Georgieve M, Rengarajan J (2015) Innate immune defense in human tuberculosis: an overview of the interaction between Mycobacterium tuberculosis and innate immune cells. J Immunol Res 2015. https://doi.org/10.1155/2015/747543, Article ID 747543, 12 pages

Siempos II, Vardakas KZ, Kopterides P, Falagas ME (2008) Adjunctive therapies for communityacquired pneumonia: a systematic review. J Antimicrob Chemother 62(4):661-668. https://doi. org/10.1093/jac/dkn283

Silva Manuel T, Correia NM (2012) Neutrophils and macrophages: the main partners of phagocyte cell systems. Front Immunol 3:174

Smith I (2003) Mycobacterium tuberculosis pathogenesis and molecular determinants of virulence. Clin Microbiol Rev 16(3):463-496

Southwick F (2007) Chapter 4: pulmonary infections. In: Infectious diseases: a clinical short course, 2nd edn. McGraw-Hill Medical Publishing Division, Blacklick, pp 104-313-4. isbn:0-07-147722-5

Stokes KL, Currier MG, Sakamoto K, Lee S, Collins PL, Plemper RK, Moore ML (2013) The respiratory syncytial virus fusion protein and neutrophils mediate the airway mucin response to pathogenic respiratory syncytial virus infection. J Virol 87(18):10070-10082

Sun K, Yajjala VK, Bauer C, Talmon GA, Fischer KJ, Kielian T, Metzge DW (2016a) Nox2derived oxidative stress results in inefficacy of antibiotics against post-influenza $S$. aureus pneumonia. J Exp Med 213(9):1851-1864

Sun K, Yajjala VK, Bauer C, Talmon GA, Fischer KJ, Kielian T, Metzger DW (2016b) Nox2derived oxidative stress results in inefficacy of antibiotics against post-influenza S. aureus pneumonia. J Exp Med 213(9):1851-1864. https://doi.org/10.1084/jem.20150514. Epub 2016 Aug 15

Tate Michelle D, Pickett DL, van Rooijen N, Brooks Andrew G, Reading Patrick C (2008) The role of neutrophils in the upper and lower respiratory tract during influenza virus infection of mice. Respir Res 9:57. https://doi.org/10.1186/1465-9921-

Tate Michelle D, Pickett Danielle L, van RN, Brooks AG, Reading Patrick C (2010) Critical role of airway macrophages in modulating disease severity during influenza virus infection of mice. J Virol 84(15):7569-7580

Taubenberger JK, Morens DM (2008) The pathology of influenza virus infections. Annu Rev Pathol 3:499-522

Trefler S, Rodriguez A, Martin-Loeches I, Sanchez V, Marin J, Llaurado M, Romeu M, Diaz E, Nogues R, Giralt M (2014) Oxidative stress in immunocompetent patients with severe community-acquired pneumonia. A pilot study. Med Intensiva 38(2):73-82

Tuberculosis (TB) World Health Organization. 16 February 2018

Tumpey et al (2005) Pathogenicity of influenza viruses with genes from the 1918 pandemic virus: functional roles of alveolar macrophages and neutrophils in limiting virus replication and mortality in mice. J Virol 79(23):14933-14944 
Ugurlu E (2016) Increased erythrocyte aggregation and oxidative stress in patients with idiopathic interstitial pneumonia. Clin Res 33(4):308-316

Vergne I, Chua J, Lee HH, Lucas M, Belisle J, Deretic V (2005) Mechanism of phagolysosome biogenesis block by viable Mycobacterium tuberculosis. Proc Natl Acad Sci U S A 102:4033-4038

Verma I, Jindal S, Ganguly NK (2014) Chapter: oxidative stress in tuberculosis. In: Book: studies on respiratory disorders 2197-7224. Humana Press, New York, pp 101-114

Vijayamalini M, Manoharan S (2004) Lipid peroxidation, vitamins C, E and reduced glutathione levels in patients with pulmonary tuberculosis. Cell Biochem Funct 22(1):19-22

Wagh V, Rajopadhye S, Mukherjee S, Urhekar A, Modi D (2016) Assessment of oxidative stress in serum of pulmonary tuberculosis patients. Int J Res Med Sci 4(8). https://doi. org/10.18203/2320-6012.ijrms20162288

Wiid I, Seaman T, Hoal EG, Benade AJ, Van Helden PD (2004) Total antioxidant levels are low during active TB and rise with Antituberculosis therapy. IUBMB Life 56(2):101-106

www.who.int/tb/challenges/hiv/07_tb_prevention_diagnosis_and_treatment_eng.pdf

Xu Y, Liu L (2017) Curcumin alleviates macrophage activation and lung inflammation induced by influenza virus infection through inhibiting the NF- $\mathrm{KB}$ signaling pathway. Influenza Other Respir Viruses 11(5):457-463

Xu X, Zhang D, Zhang H, Wolters PJ, Killeen NP, Sullivan BM, Locksley RM, Lowell CA, Caughey GH (2006) Neutrophil histamine contributes to inflammation in mycoplasma pneumonia. J Exp Med 203(13):2907-2917 